\title{
THE ASSOCIATION OF EXPOSURE TO PM 10 WITH THE QUALITY OF LIFE IN ADULT ASTHMA PATIENTS
}

\author{
MONIKA ŚCIBOR and MARTA MALINOWSKA-CIEŚLIK
}

\author{
Jagiellonian University Medical College, Kraków, Poland \\ Faculty of Health Sciences, Institute of Public Health, Department of Environmental Health
}

\begin{abstract}
Objectives: Air pollution has become a critical environmental issue, which severely threatens the well-being of asthma patients. The quality of life of these patients, when exposed to air pollutants such as particulate matter $10\left(\mathrm{PM}_{10}\right)$, has been poorly studied. The current research examined the association between the concentration of $\mathrm{PM}_{10}$ in the air and the quality of life of patients with asthma. Material and Methods: The study group consisted of 300 adult asthma patients treated in 2 allergy outpatient clinics in Kraków, who declared they would not leave the city in the 14-day study period. Daily concentrations of $\mathrm{PM}_{10}$ from air monitoring stations were recorded over a period of 2 weeks, following which the patients filled out the standardized Asthma Quality of Life Questionnaire (AQLQ) regarding the monitored period to assess the total quality of life and its 4 domains (symptoms, limitation of activity, emotional functioning and environmental stimuli). Results: The average $\mathrm{PM}_{10}$ exposure was $65.2 \mu \mathrm{g} / \mathrm{m}^{3}$ and only $30 \%$ of the patients were exposed to values of $\leq 50 \mu \mathrm{g} / \mathrm{m}^{3}$, i.e., the highest 24-h threshold value considered acceptable by the World Health Organization. The observed effect of an increased level of exposure to airborne $\mathrm{PM}_{10}$ was associated with reduced scores in AQLQ from 0.40 at the medium level to 0.46 at the high level, in comparison to the low level. The total score of the asthma-related quality of life and its domains showed poorer outcomes as the concentration of $\mathrm{PM}_{10}$ was increasing (every 0.08 pt per a $10 \mu \mathrm{g} / \mathrm{m}^{3}$ increase). Conclusions: The increase in the concentration of $\mathrm{PM}_{10}$ in the air impacts on the overall quality of life and its particular domains in people with exceptional predispositions, such as patients with bronchial asthma. Physicians taking care of asthma patients should pay special attention to the quality of patient's life in response to the course and control of that illness, in relation to air pollution. Int J Occup Med Environ Health. 2020;33(3):311-24
\end{abstract}

Key words:

quality of life, asthma, air pollution, environmental health, $\mathrm{PM}_{10}, \mathrm{AQLQ}$

\section{INTRODUCTION}

Asthma is a common chronic non-communicable disease that affects as many as 339 million people of all ages in all parts of the world, and is increasing as people adopt modern lifestyles and live in a more urbanized environment. With a predicted increase in the proportion of the world's population living in urban areas, there is likely to be a significant increase in the number of people with asthma worldwide over the next 2 decades. It is estimated that there may be an additional 100 million people with asthma by 2025 [1,2].

Asthma causes a substantial health burden to societies, reducing the quality of life, not only due to physical and psychological health, but also due to socio-economic out-

Funding: this research was supported by the National Science Centre (grant No. DEC-2011/03/B/NZ7/00644 entitled "The influence of some environmental factors on the assessment of quality of life of patients with bronchial asthma," grant manager: Monika Ścibor, M.D., Ph.D.).

Received: August 12, 2019. Accepted: January 28, 2020.

Corresponding author: Monika Scibor, Jagiellonian University Medical College, Faculty of Health Sciences, Institute of Public Health, Department of Environmental Health, Grzegorzecka 20,31-531 Kraków, Poland (e-mail: monika.scibor@uj.edu.pl). 
comes. The various estimations of its economic burden, mostly due to productivity loss, are significant. A socioeconomic analysis for Europe reported that some 625 million limited-activity days could be attributed to the effects of air pollution by ozone and particulate matter $10\left(\mathrm{PM}_{10}\right)$ alone, especially among asthma patients [3,4].

Human health may be adversely affected by long-term exposure to air pollution, even at relatively low concentrations. Epidemiological studies of the acute effects of air pollution have provided evidence for increased mortality and morbidity associated with particulate matter (PM) levels, even at low or moderate concentrations [5-7].

Many epidemiological studies use the $\mathrm{PM}_{10}$ level as an indicator of air pollution exposure. Particulate matter 10 represents the particle mass that enters the respiratory tract; moreover, it includes both coarse particles (sized $2.5-10 \mu \mathrm{m})$ and fine particles $\left(<2.5 \mu \mathrm{m}, \mathrm{PM}_{2.5}\right)$ that are considered to contribute to the health effects observed in populations living in urban environments. Particulate matter contains microscopic solids or liquid droplets that are so small that they can be inhaled and cause serious health problems. Particles of $<10 \mu \mathrm{m}$ in diameter pose the greatest threat, because they can get deep into the lungs and even the bloodstream [8]. There are numerous studies on the effects of short-term exposure to $\mathrm{PM}_{10}$, which has been used as a basis for the development of the WHO Air Quality Guidelines (AQG) and interim targets for 24-h concentrations of PM. The health risks associated with short-term exposure to $\mathrm{PM}_{10}$ produce an increase in mortality of ca. $0.5 \%$ for each $10 \mu \mathrm{g} / \mathrm{m}^{3}$ increment in the daily concentration. Therefore, a $\mathrm{PM}_{10}$ concentration of $150 \mu \mathrm{g} / \mathrm{m}^{3}$ would be expected to translate into roughly a $5 \%$ increase in daily mortality, an impact that would be of significant concern, and one for which immediate mitigation actions would be recommended [9].

Patients with asthma are one of the most at risk groups of morbidity caused by air pollution. From a mechanis- tic perspective, air pollutants cause oxidative injury to the airways, leading to inflammation, remodeling, and an increased risk of sensitization. Ambient levels of PM exacerbate existing asthma, especially by contributing to oxidative stress and allergic inflammation. There is also some evidence in support of PM as a cause of new cases of asthma in all populations, both children and adults [10]. The association between air pollution and asthma morbidity is well proven. Patients with asthma living in urban areas with high particulate pollution levels are more likely to have frequent asthma symptoms, asthma-related emergency department visits and hospitalizations than those living in areas with low pollution [11]. A systematic review and meta-analysis of cohort studies have proven the association between long-term exposure to air pollution and the incidence of asthma. Epidemiological studies have shown the impact of air pollutants on the increasing incidence of asthmatic symptoms and other adverse health effects. These results suggest a deleterious effect of ambient air pollution on the incidence of asthma in adults [12-14].

There is epidemiological evidence that asthmatic symptoms can be worsened by an increase in $\mathrm{PM}_{10}$. Exposure to air pollution contributes to asthma development, aggravations and exacerbations. Typical outdoor air pollutants that can trigger asthmatic symptoms include PM, with the severity of symptoms varying with the level of exposure. An increase in air pollutants (i.e., ozone and PM) raises the risk of both asthma development and its symptoms. Exposure to $\mathrm{PM}_{10}$ can trigger or exacerbate asthma attacks, and control is essential for proper risk management and risk communication for the exposed asthma patients, especially those living in urban environments $[15,16]$. Asthma as a chronic disease could cause problems and difficulties in everyday functioning, in psychological and mental health related to the prevalence of anxiety and depression symptoms, which impact on subjective health. A lot of asthma patients have screened posi- 
tive for depressive symptoms, and they report a worse quality of life during control visits in the outpatient allergy clinic, but it is not well confirmed by epidemiological studies. Therefore, the asthma-related quality of life (ARQL) measured by disease-specific scales should also be considered in the assessment of the social and health status of these patients [17,18]. Measurements of health among asthma patients, and also the effects of health care, must include not only an indication of changes in the frequency and severity of asthma or its symptoms, but also an estimation of self-assessed well-being. This should be assessed by measuring the changes in the patient's quality of life. The health-related quality of life (HRQL) is a broad ranging concept affected in a complex way by the person's own physical health, psychological state and mental health, the level of independence, social relationships, personal health beliefs, and the relationship to the salient features of their physical environment, including air pollutants [19].

It seems that verification of the quality of life of patients with bronchial asthma may be as useful as routine diagnostic methods that assess the disease course control.

In the asthma management and treatment process, HRQL is an important cause and outcome. In clinical practice, the assessment of the quality of life gives valuable information that can indicate areas in which the patient is most affected and needs support. It helps the physician in advising and making the best choices in patient care. Surveys have shown that differences in assessing the quality of life depend on the patient's gender and place of residence, which may indicate the groups to which therapeutic and educational efforts should be directed [20,21]. It seems that in assessing the impact of air pollution on asthma control, an analysis of the patient's quality of life should be prognostic in the treatment process.

The Asthma Quality of Life Questionnaire (AQLQ) is one of the most popular tools used to measure changes in HRQL over the course of treatment of this chronic dis- ease. The use of this questionnaire has proven that a $0.5-\mathrm{pt}$ decrease in the total score of AQLQ is a harbinger of an unfavorable course of the disease [22,23].

The HRQL in patients with asthma exposed for a long time to air pollutants, such as $\mathrm{PM}_{10}$, in the urban environment has been poorly studied. There is little evidence that $\mathrm{PM}_{10}$ reduces the quality of life in asthma patients, and could be related to the likelihood of the initial sensitization and induction of both the disease and syndromes. Only limited evidence exists on the impact of $\mathrm{PM}_{10}$ on the HRQL of patients with bronchial diseases. There is no conclusive confirmation of the unambiguously detrimental connection between the quality of life and the concentration of $\mathrm{PM}_{10}$ in the outdoor air. A pilot study conducted in 2013 in Kraków, Poland, did not significantly prove the association between $\mathrm{PM}_{10}$ concentrations in the outdoor air and the quality of life of asthma patients [24]. Since the assessment of the impact of exposure to air pollutants, such as $\mathrm{PM}_{10}$, on the quality of life of patients with bronchial asthma has not yet been the subject of a sufficient number of studies, this study gives an additional opportunity to assess and discuss this problem, in order to improve the well-being of adult asthma patients.

The objective of the study was to assess the impact of $\mathrm{PM}_{10}$ exposure on the quality of life of patients with asthma living in Kraków, and to determine whether this exposure was related to changes in their subjective health. Results of this study should be important in planning preventive programs, risk communication and patients' education to improve their well-being.

\section{MATERIAL AND METHODS}

A group of 349 adults patients ( $>18$ years old) with moderate asthma, treated in 2 allergy outpatient clinics located in Kraków, were recruited for a 2-year survey conducted in 2013-2015. These patients were with partially controlled asthma, living in Kraków, and they declared not to leave the city during 14 days of the survey. Each pa- 
tient was subjected to observation for 2 weeks. The surveyed patients with bronchial asthma were diagnosed according to the applicable standards, and they were under permanent control of a physician specializing in allergic diseases. In the study group, the recruited patients were under regular treatment in an outpatient allergy clinic. The group was fairly homogenous, i.e., it included patients with partially controlled asthma, well educated in asthma, recognizing and controlling symptoms of the disease, with a good knowledge of the types and use of medication, and regularly taking their medications.

The exposure to $\mathrm{PM}_{10}$ depended on the observation period, and the place of residence in the city district was controlled and measured.

During the first visit, the patients signed an informed consent form and agreed to participate in the survey. They were provided with the observational patient's diary and a peak flow meter, and they were trained to take measurements of the peak expiratory flow rate (PEFR) using that device.

During the second visit, after 14 days, the patients were asked to assess their quality of life using AQLQ. The questionnaire is designed to be standardized and sufficiently sensitive to detect and measure the size of any changes in the subjective health in patients with asthma, and it is a disease-specific measure of their HRQL.

The questionnaire consists of 32 questions, grouped in the following 4 domains of the quality of life:

- symptoms,

- limitation of activity,

- emotional functioning,

- environmental stimuli.

The patients provided their responses on a 7-degree scale, where "7" means no limitation and "1" means a total limitation. The total score of AQLQ is a mean of all 32 responses, and the scores in particular domains are the means for positions which they include. The quality of life was determined as a mean of all responses. This result indicated a total score of the quality of life for each patient. Thus, the quality of life for particular domains was calculated. The 4 domains differed in the number of questions, with symptoms including 12 questions, limitation of activity - 11, emotional functioning -5 , and environmental stimuli -4 .

The patient's diary was used as a next tool. Its first part was completed by a physician specializing in allergic diseases during the first visit, including the date of birth, the place of residence, education, employment, workplace, way to work, smoking, keeping pets at home, and the type of treatment applied. The second part was used for everyday completion by the patient over 2 weeks of observation. Information for each patient was obtained that pertained, inter alia, to the number of hours and places they spent outside.

For each patient, a daily concentration of $\mathrm{PM}_{10}$ on each day of observation, from all available air pollution monitoring stations of the Voivodeship Environmental Protection Inspectorate in Kraków, was reported and introduced to the database. Then, the patients were assigned to the station which was the closest to their declared outdoor place of stay in order to determine what concentration of the $\mathrm{PM}_{10}$ fraction the patients were exposed to in the survey period. A selected address for each patient was verified and then entered into Google maps. The point measured on the map was determined with the command "measure distance." Then, the locations for 3 stations of the Voivodeship Environmental Protection Inspectorate in Kraków were searched for and, at each, a point was designated by the command "distance to that place." Data about $\mathrm{PM}_{10}$ concentrations recorded by particular stations were obtained from the webpage of the Voivodeship Environmental Protection Inspectorate [25].

Overall, 349 patients were recruited, but 49 of them were eventually excluded due to leaving the city in the observation period, or not filling out/losing the patient's diary. Finally, data of 300 patients aged 20-80 years (average $53 \pm 15.3$ year) were used for the analysis, including 145 women (48.3\%) and 155 men (51.7\%). 
The study was conducted with the approval of the Bioethics Committee of the Jagiellonian University (No. KBET/167/B/2012).

\section{Statistical analyses}

All characteristics were presented as means with standard deviations (SD) or frequency with percentage distribution, respectively to the measurement scale. Results regarding the quality of life of asthma patients (the AQLQ total score and its domains) were presented using the median with quartile distribution. The 2-week average $\mathrm{PM}_{10}$ concentrations were additionally categorized with cut-offs at 30th and 70th percentiles (values of 50 and $80 \mu \mathrm{g} / \mathrm{m}^{3}$, respectively). The first cut-off at 30th percentile corresponded to the 24-h threshold guided by the WHO, while 70th percentile of the highest $\mathrm{PM}_{10}$ concentration was selected as the second cut-off. In addition, $\mathrm{PM}_{10}$ concentrations were analyzed both as continuous and categorical variables. Potential confounders, such as gender, education, smoking, employment and keeping pets at home, were controlled in the analyses.

Associations between nominal variables were verified using the $\chi^{2}$ test or the exact Fisher test, as appropriate. Differences in the age of the patients exposed to different concentrations of $\mathrm{PM}_{10}$ categories were tested using one-way ANOVA, whereby the equality of variances was verified with Levene's test and normal distribution in subgroups with the Kolmogorov-Smirnov test. Outdoor temperature differences across the $\mathrm{PM}_{10}$ categories were tested with the Kruskal-Wallis test as the assumption of variance homogeneity was not met in ANOVA. The nonparametric trend in AQLQ scores across the $\mathrm{PM}_{10}$ categories was also checked [26,27].

The potential impact of airborne $\mathrm{PM}_{10}$ on the quality of life of asthma patients (the AQLQ total score and its domains) was estimated in linear regression models with 2-week average $\mathrm{PM}_{10}$ concentrations, both as categorical and continuous variables, with a unit of $10 \mu \mathrm{g} / \mathrm{m}^{3}$, adjusted for age (in years), gender, university education (yes vs. no), smoking (yes vs. no) and the mean outdoor temperature in the measurement period. Among the variables considered as potential confounders, age, university education and temperature in the measurement period were associated with AQLQ and its domains, in a univariate analysis. Gender and smoking were a priori added to the models. The potential collinearity between $\mathrm{PM}_{10}$ concentrations and temperature was checked using the variance inflation factor (VIF = 1.86), whose values allowed for putting those variables into 1 model.

The AQLQ itself was classified into 3 categories, i.e., a good quality of life (a score of 6-7), a reduced quality of life (a score of 4-5) and a poor quality of life (a score of 1-3), where " 1 " meant good and " 3 " meant poor. The $\mathrm{PM}_{10}$ exposure level (both as a categorical and continuous variable) was used to assess its impact on the poor quality of life using ordered logistic regression models adjusted for age, age ${ }^{2}$, gender, university education, active smoking and temperature in the measurement period. The same analyses were conducted for all 4 domains, as 4 separate tests. The proportional odds assumption was verified using the Brant test. The term "age" " was added as the age variable did not comply with the proportional odds assumption.

All the tests were 2-tailed, and significance was set at $\mathrm{p}<0.05$. All the analyses were performed using STATA/ IC 13.1 software.

\section{RESULTS}

The average $\mathrm{PM}_{10}$ exposure (a mean in the 2-week period) was $65.2 \mu \mathrm{g} / \mathrm{m}^{3}$ (a range of $18.0-128.2 \mu \mathrm{g} / \mathrm{m}^{3}$ ) and only 75 patients (30\%) were exposed to values of $\leq 50 \mu \mathrm{g} / \mathrm{m}^{3}$, i.e., the 24-h threshold recommended by WHO. The $\mathrm{PM}_{10}$ measurements were equally distributed across seasons. There were no significant differences between the patients in the number of hours spent outdoors. In general, all the patients declared that they spent 2-3 h/day outdoors, on average. 
The groups of patients with various average $\mathrm{PM}_{10}$ exposure levels did not differ significantly in terms of gender, education (university vs. lower), employment or keeping pets at home. Nevertheless, the patients exposed to higher levels of $\mathrm{PM}_{10}$ were older, and the studied subgroups were significantly different in terms of smoking (Table 1). Declining trends in the total and specific domains of AQLQ were observed with the increasing levels of airborne $\mathrm{PM}_{10}$ exposure (Figure 1).
Table 2 shows the effects of categorized $\mathrm{PM}_{10}$ exposure on the ARQL adjusted for potential confounders. The observed effect of an increased level of exposure to airborne $\mathrm{PM}_{10}$ was associated with reduced scores in AQLQ from 0.40 at the medium level, as compared to the low level, to 0.46 at the high level, as compared to the low level. A similar decrease was observed in the symptoms and activity limitation domains with differences

Table 1. Characteristics of the study group of adult asthma patients over the follow-up period and exposure to $\mathrm{PM}_{10}$ levels, Kraków, Poland, 2013-2015

\begin{tabular}{|c|c|c|c|c|c|}
\hline \multirow[b]{2}{*}{ Variable } & \multicolumn{4}{|c|}{$\begin{array}{l}\text { Respondents exposed to } \mathrm{PM}_{10} \\
\qquad(\mathrm{~N}=300)\end{array}$} & \multirow[b]{2}{*}{$\mathrm{p}$} \\
\hline & total & $\begin{array}{c}\text { low level } \\
\left(\leq 50 \mu \mathrm{g} / \mathrm{m}^{3}\right) \\
(\mathrm{N}=90)\end{array}$ & $\begin{array}{c}\text { medium level } \\
\left(50.1-80 \mu \mathrm{g} / \mathrm{m}^{3}\right) \\
(\mathrm{N}=122)\end{array}$ & $\begin{array}{c}\text { high level } \\
\left(>80 \mu \mathrm{g} / \mathrm{m}^{3}\right) \\
(\mathrm{N}=88)\end{array}$ & \\
\hline Gender [n (\%)] & & & & & $0.679^{\mathrm{a}}$ \\
\hline women & $145(48.3)$ & $42(46.7)$ & $57(46.7)$ & $46(52.3)$ & \\
\hline men & $155(51.7)$ & $48(53.3)$ & $65(53.3)$ & $42(47.7)$ & \\
\hline Age [years] $(\mathrm{M} \pm \mathrm{SD})$ & $53 \pm 15.3$ & $49.5 \pm 15.0$ & $52.3 \pm 15.3$ & $57.5 \pm 14.7$ & $0.002^{b}$ \\
\hline Education $[\mathrm{n}(\%)]$ & $127(42.3)$ & $46(51.1)$ & $51(41.8)$ & $30(34.1)$ & $0.071^{\mathrm{a}}$ \\
\hline Active smoker $[\mathrm{n}(\%)]$ & $31(10.3)$ & $3(3.3)$ & $18(14.8)$ & $10(11.4)$ & $0.024^{\mathrm{a}}$ \\
\hline Current employment [n (\%)] & & & & & $0.247^{c}$ \\
\hline student & $11(3.7)$ & $3(3.3)$ & $7(5.8)$ & $1(1.1)$ & \\
\hline employed & $172(57.5)$ & $58(64.4)$ & $68(56.2)$ & $46(52.3)$ & \\
\hline unemployed & $27(9.0)$ & $8(8.9)$ & $12(9.9)$ & $7(8)$ & \\
\hline retired & $89(29.8)$ & $21(23.3)$ & $34(28.1)$ & $34(38.6)$ & \\
\hline missing & $1(0.3)$ & & & & \\
\hline Keeping pets at home $[\mathrm{n}(\%)]$ & $133(43.3)$ & $39(43.8)$ & $46(38)$ & $46(52.3)$ & $0.122^{\mathrm{a}}$ \\
\hline Temperature $\left[{ }^{\circ} \mathrm{C}\right](\mathrm{M} \pm \mathrm{SD})$ & $7.8 \pm 5.88$ & $13.8 \pm 5.06$ & $6.0 \pm 4.47$ & $4.0 \pm 2.79$ & $<0.001^{\mathrm{d}}$ \\
\hline \multicolumn{6}{|c|}{ Asthma Quality of Life Questionnaire (Me (Q1-Q3)) } \\
\hline total score & $5(4-6)$ & $6(5-6)$ & $5(4-6)$ & $5(3-6)$ & $<0.001^{\mathrm{e}}$ \\
\hline symptoms & $6(4-6)$ & $6(5-7)$ & $5(4-6)$ & $5(3-6)$ & $<0.001^{\mathrm{e}}$ \\
\hline limitation of activity & $5(4-6)$ & $6(5-6)$ & $5(4-6)$ & $4.5(3-5.5)$ & $<0.001^{\mathrm{e}}$ \\
\hline emotional functioning & $5(4-6)$ & $6(5-6)$ & $5(4-6)$ & $5(3-6)$ & $<0.001^{\mathrm{e}}$ \\
\hline environmental stimuli & $5(4-5)$ & $5(5-6)$ & $4(4-5)$ & $4(3-5)$ & $<0.001^{\mathrm{e}}$ \\
\hline
\end{tabular}



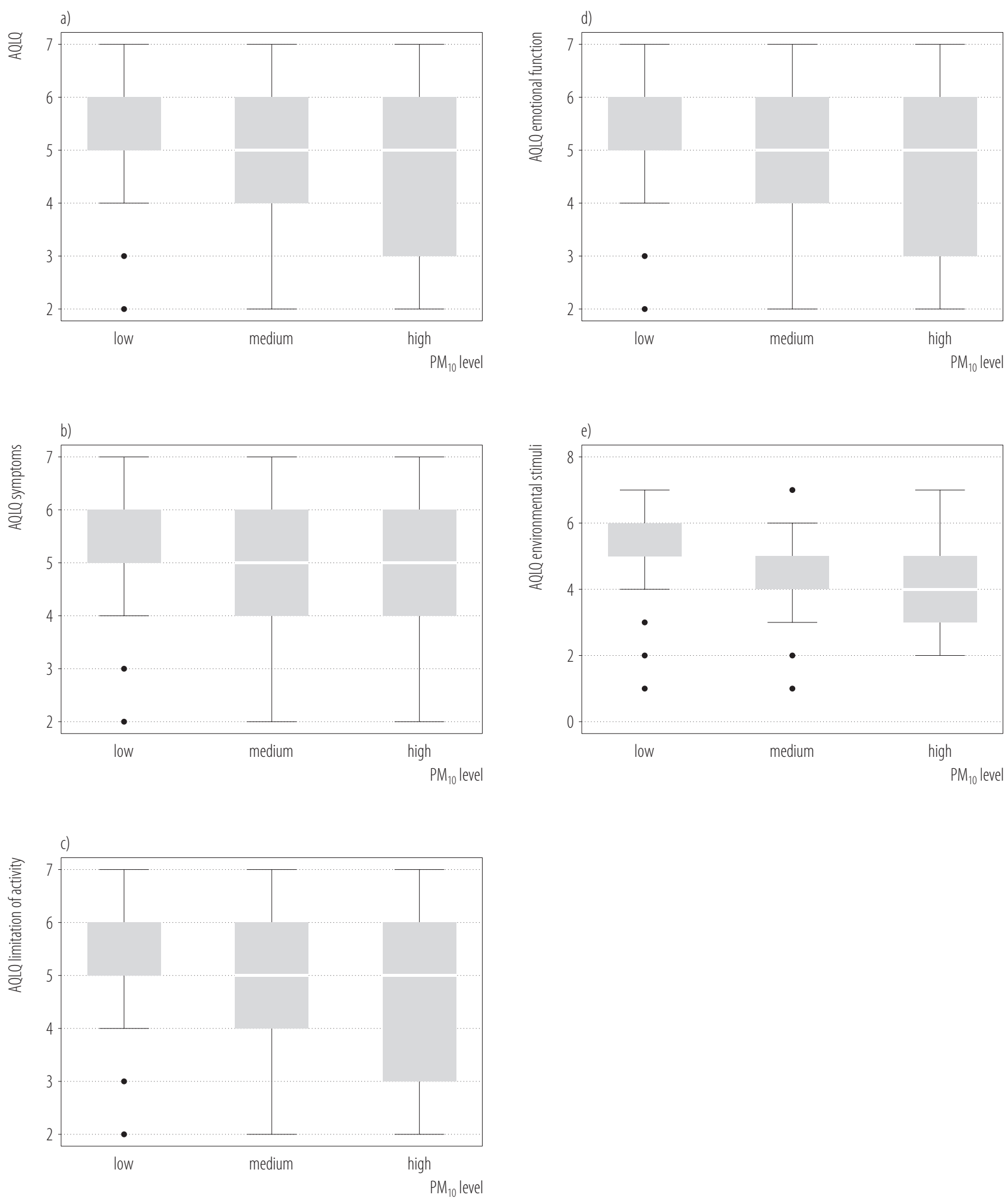

Figure 1. Distribution of a) Asthma Quality of Life Questionnaire (AQLQ) total scores and its domains: b) symptoms, c) limitation of activity, d) emotional functioning, e) environmental stimuli according to $\mathrm{PM}_{10}$ levels in adult asthma patients, Kraków, Poland, 2013-2015 
Table 2. Impact of categorized particulate matter $10\left(\mathrm{PM}_{10}\right)$ concentrations on the asthma-related quality of life and the risk of a poorer quality of life in the study group of adult asthma patients, Kraków, Poland, 2013-2015

\begin{tabular}{|c|c|c|c|c|c|c|}
\hline \multirow{3}{*}{$\begin{array}{l}\text { AQLQ and its domains/ } \\
\mathrm{PM}_{10} \text { levels }\end{array}$} & \multicolumn{6}{|c|}{ Asthma Quality of Life Questionnaire (AQLQ) score } \\
\hline & \multicolumn{3}{|c|}{ original scale* } & \multicolumn{3}{|c|}{ categorized $^{* *}$} \\
\hline & B & $95 \% \mathrm{CI}$ & $\mathrm{p}$ & $\mathrm{OR}^{\mathrm{a}}$ & $95 \% \mathrm{CI}$ & $\mathrm{p}^{\mathrm{a}}$ \\
\hline \multicolumn{7}{|l|}{ Total score } \\
\hline medium vs. low & -0.40 & $-0.76-(-0.04)$ & 0.030 & 4.29 & $1.94-9.52$ & $<0.001$ \\
\hline high vs. low & -0.46 & $-0.88-(-0.04)$ & 0.030 & 6.03 & $2.45-14.88$ & $<0.001$ \\
\hline \multicolumn{7}{|l|}{ Symptoms } \\
\hline medium vs. low & -0.37 & $-0.75-0.01$ & 0.054 & 3.76 & $1.60-8.90$ & 0.003 \\
\hline high vs. low & -0.51 & $-0.95-(-0.07)$ & 0.023 & 6.77 & $2.56-17.92$ & $<0.001$ \\
\hline \multicolumn{7}{|l|}{ Limitation of activity } \\
\hline medium vs. low & -0.29 & $-0.67-0.09$ & 0.137 & 2.11 & $0.998-4.46$ & 0.051 \\
\hline high vs. low & -0.46 & $-0.90-(-0.02)$ & 0.040 & 2.81 & $1.21-6.54$ & 0.016 \\
\hline \multicolumn{7}{|l|}{ Emotional functioning } \\
\hline medium vs. low & -0.23 & $-0.68-0.21$ & 0.304 & 2.02 & $0.97-4.24$ & 0.061 \\
\hline high vs. low & -0.41 & $-0.93-0.11$ & 0.119 & 2.37 & $1.02-5.49$ & 0.045 \\
\hline \multicolumn{7}{|l|}{ Environmental stimuli } \\
\hline medium vs. low & -0.39 & $-0.79-0.02$ & 0.063 & 1.89 & $0.94-3.79$ & 0.073 \\
\hline high vs. low & -0.40 & $-0.87-0.07$ & 0.097 & 2.45 & $1.10-5.47$ & 0.029 \\
\hline
\end{tabular}

B - regression coefficient.

* Linear regression model adjusted to age (years), gender, university education, smoking and temperature in the measurement period.

** Ordered (from "1" - good to " 3 " - poor) logistic regression model adjusted for age (years), age ${ }^{2}$, gender, university education, smoking

and temperature in the measurement period.

of 0.51 and 0.46 at the high level of exposure, compared to the low level, respectively. In terms of emotional functioning and environmental stimuli, the quality of life did not differ significantly between the medium and high levels, compared to the low level of $\mathrm{PM}_{10}$.

In order to assess the effects of $\mathrm{PM}_{10}$ exposure on the risk of poorer outcomes in AQLQ (both total and domains scores), multivariable ordinal logistic regression models were applied. It was observed that the medium levels of $\mathrm{PM}_{10}$ exposure were associated with an over 4-fold higher risk of poorer outcomes in AQLQ (OR $=4.29,95 \% \mathrm{CI}$ : 1.94-9.52), while the high exposure with a 6-fold higher risk $(\mathrm{OR}=6.03,95 \% \mathrm{CI}: 2.45-14.88)$, compared to the low levels of $\mathrm{PM}_{10}$ exposure. Similar results were seen in the symptoms domain. The risk of a poorer quality of life in terms of symptoms reached a value that was 3.76 (95\% CI: 1.60-8.90) times higher for the medium level of $\mathrm{PM}_{10}$ and 6.77 (95\% CI: 2.56-17.92) for the high level, compared to the low one. The risk of poor outcomes in terms of limitation of activity was 2.81 times higher, in emotional functioning 2.37 times higher, and in environmental stimuli 2.45 times higher (95\% CI: 1.67-6.26), but only in the high vs. low level of the $\mathrm{PM}_{10}$ concentration (Table 2).

The effects of the continuously expressed mean of the 14-day level of $\mathrm{PM}_{10}$ exposure on ARQL, adjusted for potential confounders, are shown in Table 3. All the domains of AQLQ, except environmental stimuli, present- 
Table 3. Impact of the average 14-day concentrations of $\mathrm{PM}_{10}\left(\mathrm{unit}: 10 \mu \mathrm{g} / \mathrm{m}^{3}\right.$ ) on the asthma-related quality of life and the risk of a poorer quality of life in the study group of adult asthma patients, Kraków, Poland, 2013-2015

\begin{tabular}{lcccccc}
\hline \multirow{2}{*}{$\begin{array}{c}\text { AQLQ } \\
\text { and its domains }\end{array}$} & \multicolumn{5}{c}{ Asthma Quality of Life Questionnaire (AQLQ) score } \\
\cline { 2 - 6 } & \multicolumn{5}{c}{ original scale* } & \multicolumn{4}{c}{ categorized $^{* *}$} \\
\cline { 2 - 6 } & $\mathrm{B}$ & $95 \%$ CI & $\mathrm{p}$ & OR & $95 \%$ CI & $\mathrm{p}$ \\
\hline Total score & -0.08 & $-0.13-(-0.02)$ & 0.010 & 1.28 & $1.14-1.44$ & $<0.001$ \\
Symptoms & -0.08 & $-0.14-(-0.02)$ & 0.008 & 1.29 & $1.14-1.45$ & $<0.001$ \\
Limitation of activity & -0.08 & $-0.14-(-0.02)$ & 0.009 & 1.20 & $1.07-1.35$ & 0.002 \\
Emotional functioning & -0.08 & $-0.15-(-0.01)$ & 0.029 & 1.12 & $1.002-1.26$ & 0.046 \\
Environmental stimuli & -0.07 & $-0.13-(-0.003)$ & 0.060 & 1.15 & $1.03-1.29$ & 0.013 \\
\hline
\end{tabular}

Explanations as in Table 2.

ed poorer outcomes with the growing concentration of $\mathrm{PM}_{10}$ (every $0.08 \mathrm{pt}$ for a $10 \mu \mathrm{g} / \mathrm{m}^{3}$ increase). The risk of a poorer $\mathrm{ARQL}$ was increasing with higher $\mathrm{PM}_{10}$ exposure. A $10 \mu \mathrm{g} / \mathrm{m}^{3}$ increase in $\mathrm{PM}_{10}$ was significantly associated with a $28 \%$ higher risk of poorer total AQLQ $(\mathrm{OR}=1.28$, $95 \%$ CI: 1.14-1.44) when adjusted for age, gender, university education, smoking and temperature. Similar results were observed in each domain, where a $10 \mu \mathrm{g} / \mathrm{m}^{3}$ increase in $\mathrm{PM}_{10}$ raised the risk of poorer outcomes, from $12 \%$ in emotional functioning to $29 \%$ in symptoms (Table 3). Predicted probabilities of "poor" asthma-related quality of life according to $\mathrm{PM}_{10}$ exposure in the total score of AQLQ and its domains are presented in Figure 2. As stated, the shadowed areas show a $95 \% \mathrm{CI}$ of the estimated probability.

\section{DISCUSSION}

A lower quality of life is an important predictor of hospitalization and mortality among patients with chronic obstructive pulmonary disease (COPD) and asthma [28]. Coarse PM fractions can lead to adverse responses in the lungs, triggering processes leading to hospital admissions [29]. This suggests that special consideration should be given to studying and regulating coarse particles separately from fine particles. Results of the study by Anderson et al. [30] suggest that the active component of $\mathrm{PM}_{10}$ resides mostly in the fine fraction, and this is mostly due to primary particles from combustion sources, mainly vehicles, with a contribution from secondary particles. So, the effects of coarse fractions, such as $\mathrm{PM}_{10}$, cannot be excluded, and should be analyzed [30]. There is considerable evidence linking ambient particles measured as PM with an aerodynamic diameter of $<10 \mu \mathrm{m}\left(\mathrm{PM}_{10}\right)$ to daily mortality and hospital admissions.

The adverse health impact of $\mathrm{PM}_{10}$ on the human respiratory system is well documented by studies showing associations between the concentrations of $\mathrm{PM}_{10}$ and the quantified health effects, all cause and cause-specific mortality, morbidity and life expectancy, the incidence of respiratory diseases, and exacerbation of such diseases [31,32]. The overall epidemiological evidence suggests that these adverse health effects are dependent on both exposure concentrations and the length of exposure, and that long-term exposure has stronger and more persistent cumulative effects than short-term exposure [33]. In the study by Lu et al. [34] for a standardized increment in PM with aerodynamic diameters of $<10 \mu \mathrm{m}\left(\mathrm{PM}_{10}\right)$, there is an excessive risk of mortality and morbidity in hospitalization. Short-term exposure to $\mathrm{PM}_{10}$ was found to be positively associated with increases in mortality for non-accidental causes, cardiovascular diseases and respiratory diseases [34]. The study by Zhang et al. [35] suggests that short-term exposure to outdoor air pollution may induce or exacerbate respiratory 

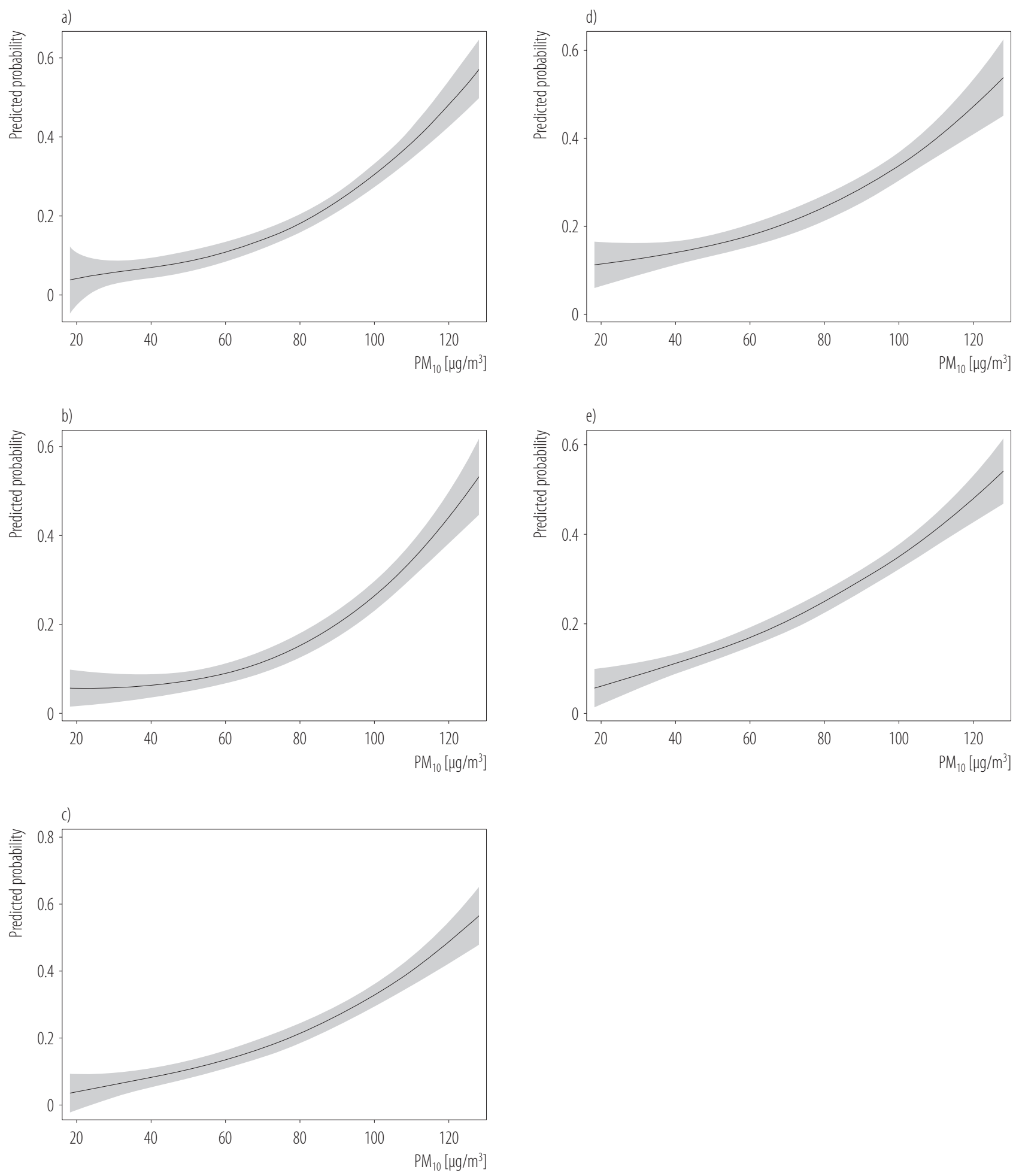

Figure 2. Predicted probabilities (with 95\% CI) of a "poor" asthma-related quality of life: a) Asthma Quality of Life Questionnaire (AQLQ) total scores and its domains: b) symptoms, c) limitation of activity, d) emotional functioning, e) environmental stimuli, according to $\mathrm{PM}_{10}$ levels in adult asthma patients, Kraków, Poland, 2013-2015 
diseases, upper respiratory tract infections, and chronic obstructive pulmonary diseases, leading to considerable medical expenditure of the affected patients.

Despite the above-mentioned epidemiological studies, the body of research investigating the influence of air pollution, including various concentrations of $\mathrm{PM}_{10}$, on the HRQL in asthma patients living in urban areas still appears insufficient. This study included an observation of the subjective health of patients with asthma, living in the urban environment of Kraków, when exposed to various concentrations of $\mathrm{PM}_{10}$. The results revealed that increased levels of $\mathrm{PM}_{10}$ were related to a lower quality of life in the surveyed patients. The total score of AQLQ and the scores for its 4 domains, such as symptoms, limitation of activity, emotional functioning and environmental stimuli, as assessed in the study, were significantly reduced by increasing concentrations of this air pollutant. A decrease in HRQL was not only caused by exceeding the highest acceptable value of $\mathrm{PM}_{10}$ exposure that was significant, but also by the degree of exceeding the norm as well. In situations where the norm was exceeded multiple times, all the domains of the patient's quality of life showed significantly lower scores. Symptoms and limitation of activity were the most affected domains of the quality of life, which decreased with every single case of exceeding the norm.

An assessment of subjective health in patients suffering from asthma has a great value in controlling the course of the disease although it is not always appreciated by physicians. The assessment of the patient's well-being consists not only of the observation of symptoms but also of the information obtained from the patient concerning his/her quality of sleep, limitation of daily activities, and experiencing negative emotions related to health threats. Based on the results of this research, one can also talk about personal exposure to environmental factors, such as dust pollution by $\mathrm{PM}_{10}$ fractions and their impact on the patient's quality of life.

Patients with asthma often report a considerable deterioration of their well-being and an intensification of symptoms during increased air pollution periods. Physicians may have problems with recognizing the reasons, i.e., whether these result from media coverage or exposure to air pollution. Popular diagnostic methods that assess the severity of bronchial asthma and its control, such as spirometry and the peak flow meter, do not ensure a full assessment of the patients' health and well-being. There are certain health-related tools to measure the quality of life in asthma patients, including sleeping disorders, limitation of activity, emotional and mental health problems, anxiety, mood swings, and depression symptoms [19,36,37]. Once applied in this study, AQLQ proved to be a good standardized tool, sensitive in detecting changes in subjective health, and in measuring asthma-specific problems related to the patient's quality of life [22,23].

The quality of life is an important issue for the contemporary understanding and assessment of the health status of patients with chronic diseases. Thus, it is important to identify and evaluate various factors which interact with and impact on their quality of life. The environmental hazards, such as air pollutants, belong to these factors. One of the goals in asthma management is to ensure an individual patient that the burden of his/her disease is controlled and limited, and that the patient is still able to have the best possible quality of life. Therefore, taking into consideration the outdoor air quality, including the $\mathrm{PM}_{10}$ concentration levels, in planning preventive and educational measures addressed to these patients, in order to improve their well-being, seems both valuable and useful [19,38]. This study had some methodological limitations regarding the assessment of individual exposure to $\mathrm{PM}_{10}$. The individual exposure to $\mathrm{PM}_{10}$ was assessed based on records for the district of the city which was the closest to the patient's place of residence. Mobile dust monitors seem to be a more precise method of evaluating $\mathrm{PM}_{10}$ exposure. However, due to their technical limitations and difficulties in patients' compliance, the use of mobile dust monitors was not possible. 


\section{CONCLUSIONS}

The results suggest that in order to attain the management goals in asthma treatment, the quality of life should be measured next to lung function, especially in the period of air pollution and exposure to $\mathrm{PM}_{10}$ in the patient's place of residence and in the surrounding urban environment. Brief and short self-administered instruments such as AQLQ provide a good opportunity to identify the patients at risk, who could benefit more from preventive interventions and patient education.

The study has shown that any exposure to increased values of $\mathrm{PM}_{10}$ impacts on both the overall quality of life and its specific domains among patients with bronchial asthma. Being aware that there is no minimal level of safe exposure to $\mathrm{PM}_{10}$, physicians should pay special attention to their patients' quality of life in relation to air pollution, especially in places where high or medium concentrations of $\mathrm{PM}_{10}$ occur during many days over long periods. This should be remembered by both physicians and patients.

It seems important to educate patients about the sources of air pollutants, such as $\mathrm{PM}_{10}$, factors influencing concentration values, and the possible strategies of reducing their individual exposure and susceptibility.

\section{REFERENCES}

1. Bousquet J, Khaltaev N, editors. Global surveillance, prevention and control of chronic respiratory diseases: a comprehensive approach. Geneva: The Organization; 2007.

2. Masoli M, Fabian D, Holt S, Beasley R. The global burden of asthma: executive summary of the GINA. Dissemination Committee Report. Allergy. 2004;59:469-78, https://doi.org/ 10.1111/j.1398-9995.2004.00526.x.

3. Global Asthma Network [Internet]. Auckland: The Organization; 2018 [cited 2019 Jul 1]. The Global Asthma Report 2018. Available from: http:/www.globalasthmareport.org/.

4. Nam K-M, Selin NE, Reilly JM, Platsev S. Measuring Welfare Loss Caused by Air Pollution in Europe: A CGE Analy- sis. Energy Policy. 2010;38(9):5059-71,https://doi.org/10.1016/ j.enpol.2010.04.034.

5. Dockery DW, Pope CA. Acute Respiratory Effects Of Particulate Air Pollution. Ann Rev Public Health. 1994;15:107-32, https://doi.org/10.1146/annurev.pu.15.050194.000543.

6. Pope CA, Dockery DW, Schwartz J. Review of epidemiological evidence of health effects of particulate air pollution. Inhal Toxicol. 1995;7:1-18, https://doi.org/10.3109/089 58379509014267.

7. Li N, Peng XW, Zhang BY. Relationship between air pollutant and daily hospital visits for respiratory diseases in Guangzhou: a time-series study. J Environ Health. 2009;26(12):1077-80.

8. United States Environmental Protection Agency [Internet]. Washington, DC: The Agency; 2019 [cited 2019 Jul 1]. Available from: https://www.epa.gov/pm-pollution.

9. World Health Organization, Occupational and Environmental Health Team [Internet]. Geneva: The Organization; 2006 [cited $2019 \mathrm{Jul}$ 1]. Air quality guidelines for particulate matter, ozone, nitrogen dioxide and sulfur dioxide. Global update 2005. Summary of risk assessment. Available from: https://apps.who.int/iris/handle/10665/69477.

10. Guarnieri M, Balmes JR. Outdoor air pollution and asthma Lancet. 2014;383(9928):1581-92, https://doi.org/10.1016/S01406736(14)60617-6.

11. Meng YY, Rull RP, Wilhelm M, Lombardi C, Balmes J, Ritz B. Outdoor air pollution and uncontrolled asthma in the San Joaquin Valley, California. J Epidemiol Community Health. 2010;64:142-7, https://doi.org/10.1136/jech.2009.083576.

12. Anderson HR, Favarato G, Atkinson RW. Long-term exposure to air pollution and the incidence of asthma: meta-analysis of cohort studies. Air Qual Atmos Health. 2013;6(1):4756, https://doi.org/10.1007/s11869-011-0144-5.

13. Li N, Hao M, Phalen R, Hinds W, Nel A. Particulate air pollutants and asthma: A paradigm for the role of oxidative stress in PM-induced adverse health effects. Clin Immunol. 2003;109(3):250-65, https://doi.org/10.1016/j.clim.2003. 08.006 . 
14. Jacquemin B, Siroux V, Sanchez M, Carsin AE, Schikowski T, Adam M, et al. Ambient Air Pollution and Adult Asthma Incidence in Six European Cohorts (ESCAPE). Environ Health Perspect. 2015;123(6):613-21, https://doi.org/10.1289/ehp.1408206.

15. Annesi-Maesano I. Environmental risk factors for asthma. In: Akdis CA, Agache I, editors. Global Atlas of Asthma the European Academy of Allergy and Clinical Immunology [Internet]. Zurich: European Academy of Allergy and Clinical Immunology; 2013 [cited 2019 Jul 1]. Available from: http:/ www.eaaci.org/GlobalAtlas/Global_Atlas_of_Asthma.pdf.

16. Donaldson K, Gilmour MI, MacNee W. Asthma and $\mathrm{PM}_{10}$. Respir Res. 2000;1:12-5, https://doi.org/10.1186/rr5.

17. Mancuso CA, Peterson M, Charlson ME. Effects of Depressive Symptoms on Health-Related Quality of Life in Asthma Patients. Gen Intern Med. 2000;15:301-10, https://doi. org/10.1046/j.1525-1497.2000.07006.x.

18. Kullowatza A, Kanniessb F, Dahmec B, Magnussenb H, Ritz T. Association of depression and anxiety with health care use and quality of life in asthma patients. Respir Med. 2007;101:638-44, https://doi.org/10.1016/j.rmed.2006.06.002.

19. World Health Organization, Division of Mental Health and Prevention of Substance Abuse [Internet]. Geneva: The Organization; 1997 [cited 2019 Jul 1]. WHOQOL: measuring quality of life. Available from: https://apps.who.int/iris/ handle/10665/63482.

20. Hesselink AE, van der Windt DA, Penninx BW, Wijnhoven HA, Twisk JW, Bouter LM, et al. What predicts change in pulmonary function and quality of life in asthma or COPD? J Asthma. 2006;43(7):513-9, https://doi.org/10.1080/027709 0060085695.

21. Tsiligianni IG, Kocks JWH, Tzanakis NE, Siafakas NM, van der Molen T. Factors that influence disease specific quality of life or health status in patients with COPD. A review and meta-analysis of Pearson correlations. Prim Care Respir J. 2011;20(3):257-68, https://doi.org/10.4104/pcrj.2011.00029.

22. Juniper EF, Guyatt GH, Epstein RS, Ferrie PJ, Jaeschke R, Hiller TK. Evaluation of impairment of health related quality of life in asthma: development of a questionnaire for use in clinical trials. Thorax. 1992;47(2):76-83, https://doi. org/10.1136/thx.47.2.76.

23. Juniper EF, Guyatt GH, Ferrie PJ, Griffith LE. Measuring Quality of Life in Asthma. Am Rev Respir Dis. 1993;147(4): 832-8, https://doi.org/10.1164/ajrccm/147.4.832.

24. Ścibor M, Balcerzak B, Czarnecka Ż, Malinowska-Cieślik M. [Assessment of life quality in patients with bronchial asthma residing in Krakow in the areas of varying concentrations of particulate matter $\left(\mathrm{PM}_{10}\right)$ ]. Environ Med. 2015;18(1):45-53. Polish.

25. [Małopolskie - system monitoringu jakości powietrza] [Internet]. Kraków: Voivodeship Environmental Protection Inspectorate; 2014 [cited 2019 Jul 1]. Available from: http:// monitoring.krakow.pios.gov.pl. Polish.

26. Cuzick J. A Wilcoxon-type test for trend. Statist Med. 1985;4:87-90, https://doi.org/10.1002/sim.4780040112.

27. Altman DG. Practical statistics for medical research. London: Chapman and Hall; 1991. p. 215-7, https://doi. org/10.1002/sim.4780101015.

28. Fan VS, Curtis JR, McDonell MB, Fihn SD. Using quality of life to predict hospitalization and mortality in patients with obstructive lung diseases. Chest. 2002;122(2):429-36, https:// doi.org/10.1378/chest.122.2.429.

29. Brunekreef B, Forsberg B. Epidemiological evidence of effects of coarse airborne particles on health. Eur Respir J. 2005;26(2):309-18, https://doi.org/10.1183/09031936.05.000 01805 .

30. Anderson HR, Bremner SA, Atkinson RW, Harrison RM, Walters S. Particulate matter and daily mortality and hospital admissions in the west midlands conurbation of the United Kingdom: association with fine and coarse particles, black smoke and sulphate. Occup Environ Med. 2001;58(1):50410, https://doi.org/10.1136/oem.58.8.504.

31. Orru H, Maasikmets M, Lai T, Tamm T, Kaasik M, Kimmel V, et al. Health impacts of particulate matter in five major Estonian towns: main sources of exposure and local differences. Air Qual Atmos Health. 2011;4:247-58, https:// doi.org/10.1007/s11869-010-0075-6. 
32. Janssen NAH, Fischer P, Marra M, Ameling C, Cassee FR. Short-term effects of PM2.5, $\mathrm{PM}_{10}$ and PM2.5-10 on daily mortality in the Netherlands. Sci Total Environ. 2013;463-4: 20-6, https://doi.org/10.1016/j.scitotenv.2013.05.062.

33. Pope CA 3rd. Mortality effects of longer term exposures to fine particulate air pollution: review of recent epidemiological evidence. Inhal Toxicol. 2007;19:33-8, https://oi. org/10.1080/08958370701492961.

34. Lu F, Xu D, Cheng Y, Dong S, Guo C, Jiang X, et al. Systematic review and meta-analysis of the adverse health effects of ambient PM2.5 and $\mathrm{PM}_{10}$ pollution in the Chinese population. Environ Res. 2015;136:196-204, https://doi.org/ 10.1016/j.envres.2014.06.029.

35. Zhang H, Niu Y, Yao Y, Chen R, Zhou X, Kan H. The impact of ambient air pollution on daily hospital visits for various respiratory diseases and the relevant medical expenditures in Shanghai, China. Int J Environ Res Public Health. 2018;15(3):425, https://doi.org/10.3390/ijerph15030425.

36. Breslow L. A quantitative approach to the World Health Organization definition of health: physical, mental and social well-being. Int J Epidemiol. 1972;1(4):347-55, https://doi. org/10.1093/ije/1.4.347.

37. Jadad AR, O'Grady L. How should health be defined? BMJ. 2008;337:a2900, https://doi.org/10.1136/bmj.a2900.

38. World Health Organization, Chronic Respiratory Diseases and Arthritis Team and WHO/WAO Meeting on the Prevention of Allergy and Allergic Asthma [Internet]. Geneva: The Organization; 2003 [cited 2019 Jul 1]. Prevention of allergy and allergic asthma. Available from: https://apps.who.int/ iris/handle/10665/68361.

This work is available in Open Access model and licensed under a Creative Commons Attribution-NonCommercial 3.0 Poland License - http://creativecommons.org/ licenses/by-nc/3.0/pl/deed.en. 